\title{
Short- and Midterm Follow-up Results of Valvuloplasty with Balloon Catheter for Congenital Aortic Stenosis
}

\author{
Carlos A. C. Pedra, Simone R. F. Pedra, Sérgio L. N. Braga, César A. Esteves, Samuel M. Moreira, \\ Magali A. dos Santos, leda J. Bosisio, Maria Aparecida P. Silva, Patrícia F. Elias, \\ Maria Virgínia T. Santana, Valmir F. Fontes
}

São Paulo, SP - Brazil

\begin{abstract}
Objective - To report short and midtem follow-up results of balloon aortic valvuloplasty to treat congenital aortic stenosis.
\end{abstract}

Methods - Seventy-five patients (median age: 8 years) underwent the procedure through the retrograde femoral or carotid route.

Results - The procedure was completed in 74 patients (98.6\%). The peak-to-peak systolic gradient dropped from $79.6 \pm 27.7$ to $22.3 \pm 17.8 \mathrm{mmHg}(P<0.001)$, the left ventricular systolic pressure dropped from 164+39.1 to $110 \pm 24.8 \mathrm{mmHg}(P<0.001)$, and the left ventricular end diastolic pressure dropped from $13.3 \pm 5.5$ to $8.5 \pm 8.3$ mmHg $(P<0.01)$. Four patients $(5.3 \%)$ died due to the procedure. Aortic regurgitation (AoR) appeared or worsened in 27/71 (38\%) patients, and no immediate surgical intervention was required. A mean follow-up of $50 \pm 38$ months was obtained in 37 patients. Restenosis and significant AoR were observed in $16.6 \%$ of the patients. The estimates for being restenosis-free and for having significant AoR in 90 months were $60 \%$ and $50 \%$, respectively.

Conclusion - Aortic valvuloplasty was considered the initial palliative method of choice in managing congenital aortic stenosis, with satisfactory short- and midterm results.

Key words: congenital aortic stenosis, aortic valvuloplasty, interventional catheterization

Instituto Dante Pazzanese de Cardiologia e Hospital do Coração da Associação Sanatório Sírio, São Paulo.

Mailing address: Carlos A. C. Pedra - Instituto Dante Pazzanese de Cardiologia Av. Dr. Dante Pazzanese, 500 - 04012-180 - São Paulo, SP, Brazil

E-mail: cacpedra@uol.com.br

English version by Stela Maris C. e Gandour
Aortic stenosis accounts for 5-6\% of the congenital heart diseases and clearly predominates in the male sex ${ }^{1}$. The presence of commissural fusion with a variable thickening and a reduction in the mobility of the valvular leaflets are almost constant anatomic findings ${ }^{1}$. Association with a bicuspid valve is also frequent ${ }^{1}$. Longitudinal studies about the natural history of the disease suggest that the intervention is indicated in the presence of severe stenosis, which is defined by clinical, electrocardiographic, and hemodynamic findings ${ }^{2}$. Surgical valvulotomy has always been the classic therapeutic method considered the gold standard. With the evolution of interventional techniques from the mid 1980s onwards, balloon valvuloplasty started to be used in the initial treatment of aortic stenosis in different age groups with satisfactory short- and midterm results ${ }^{3-29}$. We report the experience of the teams at the Instituto Dante Pazzanese and the Hospital do Coração with this type of approach.

\section{Methods}

Valvuloplasty was performed in conditions of severe stenosis, classically defined by the presence of a peak-topeak systolic gradient ${ }^{30,31}$ detected in the catheterization room or derived from echocardiography ${ }^{32,33}>70 \mathrm{mmHg}$, or, when electrocardiographic alterations (of the T wave or ST segment) or clinical symptoms (dizziness and syncope with no other causes; significant chest pain) were present, a peak-to-peak systolic gradient $>50 \mathrm{mmHg}$. The procedure was also performed in neonates or young infants (between 1 and 6 months of age) with aortic stenosis and severe ventricular dysfunction, independent of the transvalvular gradient, because in that situation that gradient is underestimated due to the low systemic cardiac output. Valvuloplasty was contraindicated in the presence of moderate and severe aortic regurgitation, of other associated intracardiac lesions requiring immediate surgery, and of a hypoplastic left ventricle requiring palliative surgery, within a therapeutic 
univentricular algorithm ${ }^{34-36}$. Written consent was obtained from the patients or their guardians after a detailed explanation about the procedure.

From May 1986 to July 2001, 75 patients (53 males) underwent balloon valvuloplasty. Their ages ranged from 5 days to 31 years (mean of $7.7 \pm 6.2$; median of 8 ) and their weights ranged from 1.1 to $85 \mathrm{~kg}$ (mean of $28.7 \pm 21.8$; median of 30$)$. Nine patients $(12 \%)$ were neonates, none of whom were receiving infusion of prostaglandins, and $8(10.6 \%)$ were 1 to 6 months old. Associated lesions were: pulmonary valve stenosis, 2 patients in whom pulmonary valvuloplasty was performed in the same session of catheterization; small muscular interventricular communication with no repercussions, 3 patients; fibroelastosis, 4 patients; previous surgery for correction of coarctation of the aorta, 3 patients; native coarctation of the aorta, 2 patients undergoing aortoplasty in the same procedure; small interatrial communication, 1 patient; and a small persistent ductus arteriosus, 1 patient undergoing occlusion of the defect in the same session according to previously described protocols ${ }^{37}$. Fifty $(66 \%)$ patients had bicuspid aortic valve, but none had unicommissural valve.

The technique of valvuloplasty comprised general anesthesia with orotracheal intubation for all age groups. In some cases, conscious sedation was used in young adults. The carotid route was used in 11 of the 17 patients younger than 6 months (one by puncture, 10 by arterial dissection), and the classic retrograde femoral route was used in the remaining patients. Carotid dissection was performed by a cardiac or vascular surgeon. The vessel was opened with a small longitudinal incision, which allowed the progression of a guidewire followed by a 5-Fr low-profile introducer. After arterial puncture or dissection, placement of the intro- ducers, and systemic heparinization (50-150 U/kg), all patients underwent angiographic and pressure studies. Left catheterization was performed with a catheter with a terminal orifice, and the pressure pullback from the left ventricular tip to the aorta was recorded. Simultaneous pressures were obtained in some cases, using the lateral arm of the introducer and smaller 1-Fr profile catheters. Aortography was performed in either the left anterior oblique or the right anterior oblique projection, or both, with a pig-tail or angiographic catheter (fig. 1). Left ventriculography was performed in elongated axial or right anterior oblique $\left(20-30^{\circ}\right)$ projection, or both (fig. 2). The degree of aortic regurgitation was assessed according to the classification of Selles modified by Moore et $\mathrm{al}^{22}$. The degree of thickening of the valvular leaflets was classified as mild, moderate, and severe according to the already published protocols ${ }^{22}$. Several catheters and guidewires were used to enter the left ventricle, going through the stenotic orifice. For dilation, a long guidewire $(0.035 \% / 260 \mathrm{~cm}$, preferably extra-stiff) was advanced to the left ventricle through a catheter with a terminal orifice, and cautiously maintained close to the ape ${ }^{30,31}$. A large loop in the extremity of the guidewire was previously molded to avoid myocardial damage (figs. 1 and 3). Over this guidewire, the preselected balloon catheter was placed in the region of the ring and manually inflated with contrast medium diluted in a saline solution. With the balloon pullback to the aorta during its filling, a constant movement was performed to push the balloon to provide greater stability to its position, mainly when the femoral route was used. The classic hourglass image was obtained during the balloon filling, with its disappearance after total expansion of the balloon (figs. 1 and 3 ). The filling and emptying time (1 cycle) did not exceed 10 seconds. One to 3 cycles were

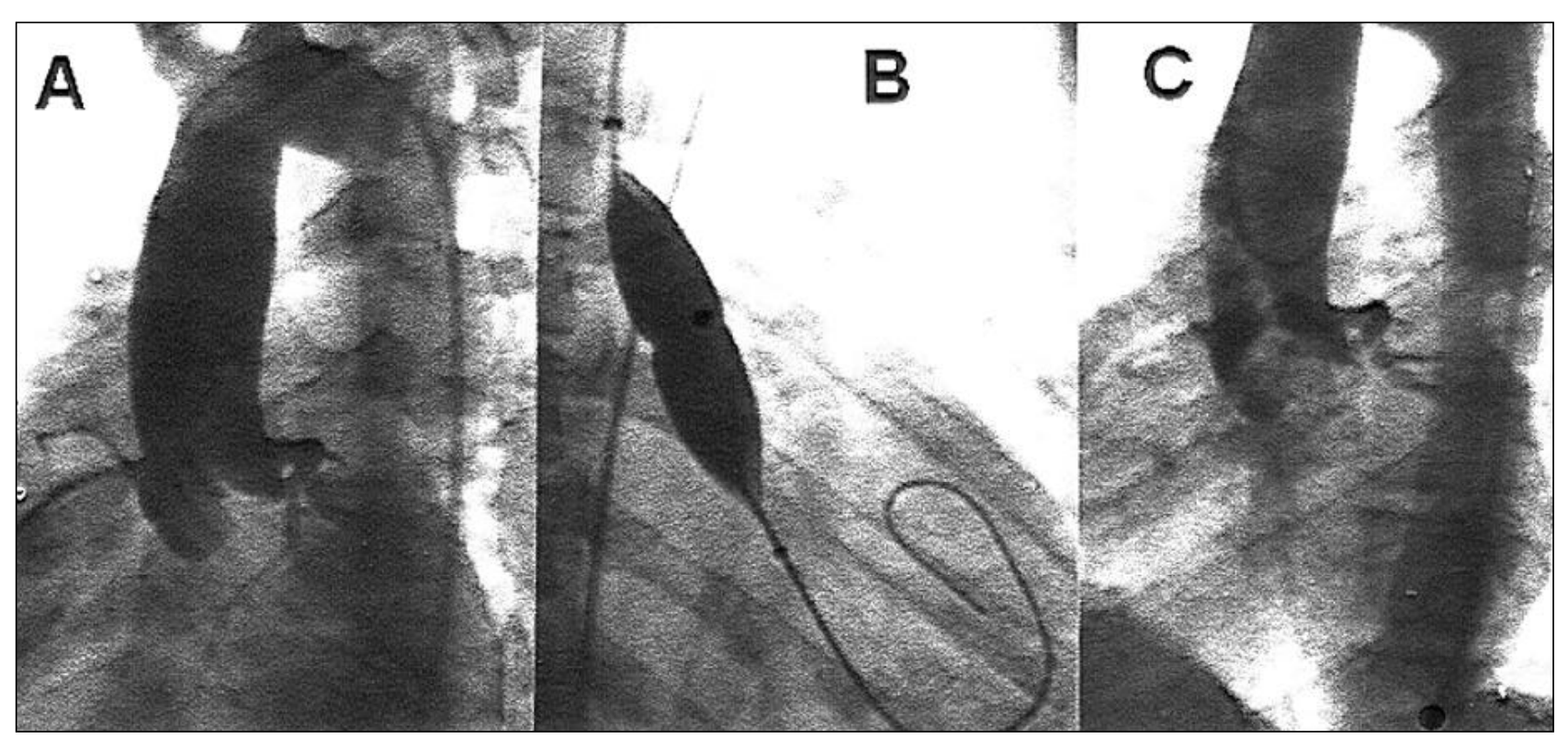

Fig. 1 - Aortography before and after dilation. A) aortography in left anterior oblique projection showing the reduced and cup-shaped opening of the aortic valve leaflets, with mild dilation of the ascending aorta and no aortic insufficiency prior to dilation; B) the balloon catheter can be seen in the region of the aortic ring forming a slight waist in the balloon, and a large loop can be seen in the distal extremity of the guidewire to avoid myocardial damage; C) enlargement of the contrast medium column is observed as a result of widening of the stenotic orifice after dilation, without induction of aortic regurgitation. 


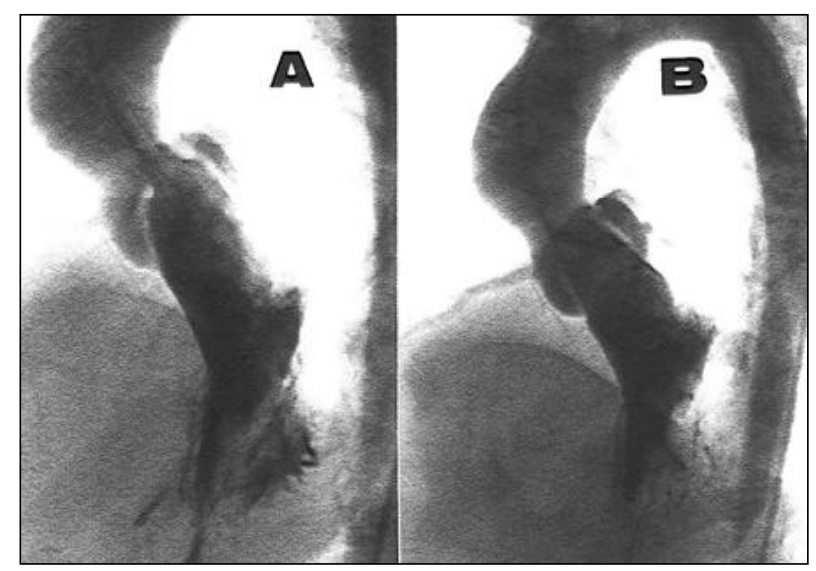

Fig. 2 - Ventriculography in elongated axial projection before and after dilation. A) marked ventricular hypertrophy and hyperkinetic contraction can be seen. The aortic ring dimensions have been preserved. Significant thickening, cup-shaped opening, and eccentric orifice have been observed in the leaflets. Poststenotic dilation in the ascending aorta is evident; B) a clear improvement in the valvular orifice opening resulting from an increase in the right leaflet incursion is seen.

performed during the procedure. In the last 4 patients older than 10 years, in whom the retrograde femoral route was used, adenosine (200-500 mcg/kg for children and 6-12 mg for adults) was intravenously administered right before balloon inflation to induce temporary bradycardia/asystole and to allow greater stability of the balloon in the outflow tract ${ }^{38}$. The diameter of the aortic ring was measured in the hemodynamic room through the digital method with correction of the magnification. The choice of the balloon followed the criterion of 80 to $100 \%$ of the diameter of the valvular ring with variable lengths, according to the patient's age and availability of the balloon catheters. In adolescent or adult patients with a large diameter aortic ring (> 20-25 mm), the double-balloon technique was used. The sum of the diameters of the 2 balloons was approximately 1.3 times the diameter of the ring ${ }^{39,40}$. In the last 5 years, low-profile balloon catheters (Tyshak II, Numed, Canada) have been preferred. The short-term results were assessed through new hemodynamic and angiographic studies obtained through similar form and conditions to the basal ones, right after the end of the procedure (figs. 1 and 4). Success in reducing the transvalvular gradient was defined as $>50 \%$. If the gradient did not decrease to less than $50 \mathrm{mmHg}$, in the absence of moderate/severe aortic regurgitation, additional increments in the diameter of the balloon were performed until the maximal balloon/ring ratio of 1.2 was reached. After finishing the procedure, hemostasis was obtained with manual compression in the femoral region. In patients undergoing dilation through the carotid artery route, that vessel was surgically repaired with 1 to 3 simple stitches with ultrathin mono-nylon thread.

After extubation, in the catheterization room, the patients were transferred to a recovery unit for routine monitoring. Echocardiography, chest radiography, and electrocardiography were performed. The magnitude of aortic insufficiency was defined by echocardiography according to the following various criteria: relation between the jet of insufficiency and the aortic ring (more common); presence of reverse flow in the descending aorta; and measurement of the pressure half time (PHT) ${ }^{41}$. After hospital discharge, clinic visits were performed according to the recommendations of the cardiologist 3 months after the procedure, and, then, every 6 to 12 months. At these visits, echocardiography, chest radiography, and electrocardiography were routinely performed. In the beginning of the study, 9 patients underwent control catheterization $13.5 \pm 5.4$ months after valvuloplasty.

Restenosis was arbitrarily defined by the presence of a gradient $>75 \mathrm{mmHg}$ on echocardiography (maximal instantaneous gradient) or above $50 \mathrm{mmHg}$ on catheterization (peak-to-peak gradient). These patients were referred for surgical reintervention or new valvuloplasty when no significant aortic regurgitation existed. Patients with severe

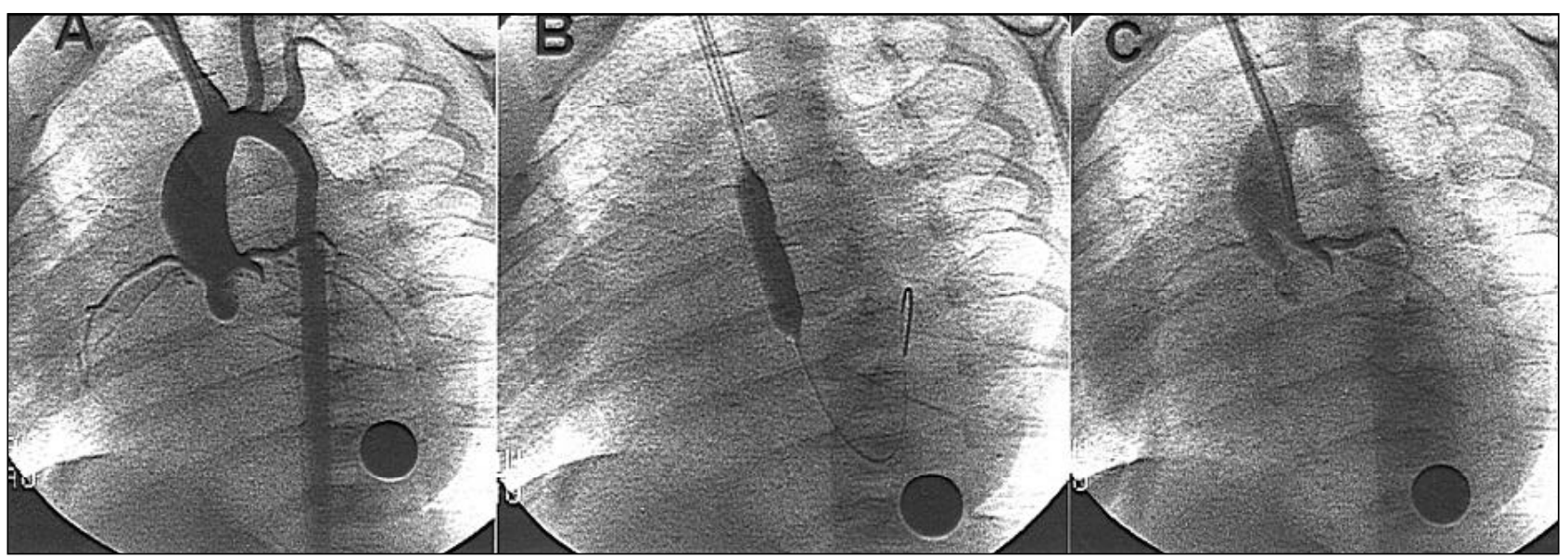

Fig. 3 - Valvuloplasty through the carotid route in the neonatal period. A) aortography in left anterior oblique projection showing the reduced and cup-shaped opening of the aortic valve leaflets. The bulging contour of the coronary arteries suggest ventricular dilation due to dysfunction already detected on previous echocardiography. In these cases, no ventriculography was performed prior to dilation; B) the balloon catheter can be seen in the region of the aortic ring with formation of a slight waist in the balloon. The sheath introduced through the carotid artery is close to the extremity of the balloon catheter, providing greater stability. The guidewire used for entrance and maintenance of the position in the left ventricle was a coronary steerable guidewire; C) enlargement of the contrast medium column is observed as a result of widening of the stenotic orifice after dilation, without induction of aortic insufficiency. 


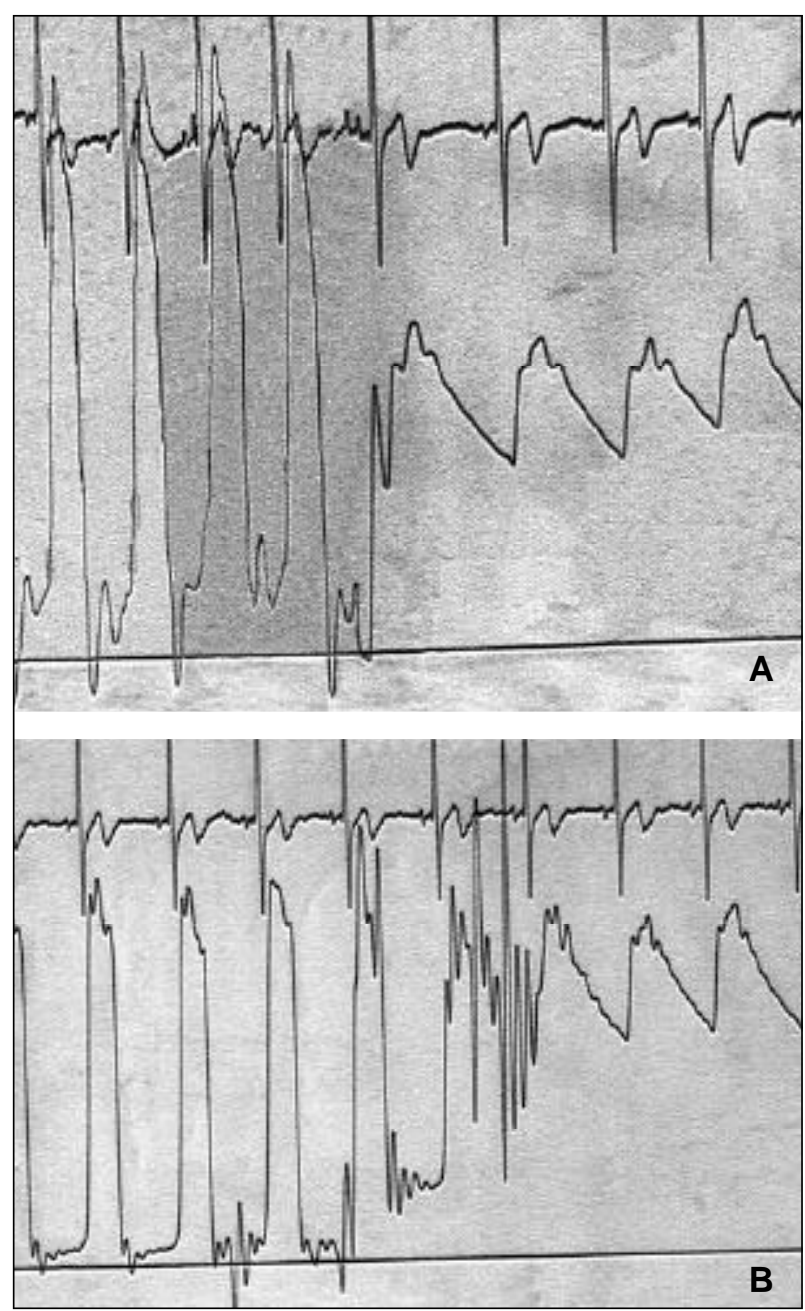

Fig. 4 - Pressure curves before and after the procedure. A) left ventricle: 180/18 mmHg; Aorta: 100/60 mmHg; B) left ventricle: $140 / 10 \mathrm{mmHg}$; Aorta: $120 / 75 \mathrm{mmHg}$. The curve pattern in the aorta does not have great amplitude, suggesting that no significant aortic regurgitation exists.

aortic regurgitation (defined on echocardiography accompanied or not by cardiac catheterization) were referred for surgical procedures (valvular replacement) usually when an enlargement in the cardiac area was observed on chest radiography or an increase in the end systolic volume was observed on echocardiography ${ }^{42}$. Asymptomatic patients not using medication, without restenosis, and with up to moderate aortic regurgitation were defined as free of events.

The values were expressed as mean \pm 2 standard deviations or median and variation according to the normality of data distribution. The variation in systolic gradient and in left ventricular systolic pressure was assessed with the paired Student $t$ test. The times of the procedure were compared with the nonpaired Student $t$ test. The search for predictors for the immediate drop in the systolic gradient, restenosis, and occurrence of aortic regurgitation was performed through logistic regression techniques. Survival curves (Kaplan-Meier) were used to estimate the rates of event-free patients. The differences between the KaplanMeier curves were assessed with the log-rank test. The value of $\mathrm{P}<0.05$ was adopted as statistically significant.

\section{Results}

The procedure was successfully completed in 74 (98.6\%) of the 75 patients. Arterial access was difficult in a neonate, culminating with severe lesion to the femoral artery, which resulted in intense bleeding and death in the catheterization room.

In the 74 remaining patients, the relation between the diameter of the balloon used and the aortic ring ranged from 0.80 to 1.20 (mean of $0.97 \pm 0.1$ ). A single balloon was used in 57 procedures, balloons with sequential increments were used in 12 procedures, and a double balloon was used in 5 patients. The following decreases were observed in the catheterization laboratory: in the peak-to-peak systolic gradient from $79.6 \pm 27.7$ to $22.3 \pm 17.8 \mathrm{mmHg}(\mathrm{P}<0.001)$; in the left ventricular systolic pressure from $164 \pm 39.1$ to 110 $\pm 24.8 \mathrm{mmHg}(\mathrm{P}<0.001)$; and in the left ventricular end diastolic pressure from $13.3 \pm 5.5$ to $8.5 \pm 8.3 \mathrm{mmHg}(\mathrm{P}<0.01)$ after dilation. Two patients with initial gradients greater than $100 \mathrm{mmHg}$ maintained residual gradients between 50 and $60 \mathrm{mmHg}$; despite the greater than $50 \%$ reduction in the gradient and use of a balloon with the greatest possible diameter for the ring, the aortic ring was slightly hypoplastic for age. No other factor influenced the immediate drop in the systolic gradient, including the following: age, weight, presence and degree of aortic regurgitation prior to the procedure, left ventricular systolic pressure, balloon-ring ratio, and thickening degree of the leaflets.

The procedure took less time when the carotid route was used $(2.5 \pm 1.1$ vs $1.5 \pm 0.5$ hour; $\mathrm{P}<0.02)$ and more time when the double balloon technique was used $(3.5 \pm 1.2$ hours; $\mathrm{P}<0.03)$. Three patients died right after the procedure, resulting in an immediate mortality rate of $5.3 \%(4 / 75)$, including the case in which the procedure was not completed. Of the 3 patients who died after the dilation, 1 was a neonate $(2.1 \mathrm{~kg})$ with severe ventricular dysfunction, who did not tolerate the manipulation of the catheters and guidewires and culminated with asystolia that could not be reversed with resuscitation maneuvers. The second patient was a 6-month-old infant who also had subaortic stenosis and had previously undergone surgical correction for coarctation of aorta. Balloon imprisonment in the femoral artery occurred after dilation, which required surgical removal and evolved to cardiogenic shock and multiple organ failure due to previous severe ventricular dysfunction. The third was an 8-year-old child with dissection and acute occlusion of the left coronary artery due to the inadequate manipulation of a straight-tip guidewire (0.035", Tefloncoated, Cook) used for entering the left ventricle.

Of the remaining 71 patients, aortic regurgitation appeared, or worsened, if it already existed, in $27(38 \%)$ patients (fig. 5). In 4 (5.6\%) patients, the aortic insufficiency was considered severe, but it did not require immediate cardiac surgery. In a multivariable analysis, no predictors of appearance or worsening of immediate aortic regurgitation were identified. The following variables were studied: age, weight, presence and degree of previous aortic regurgita- 


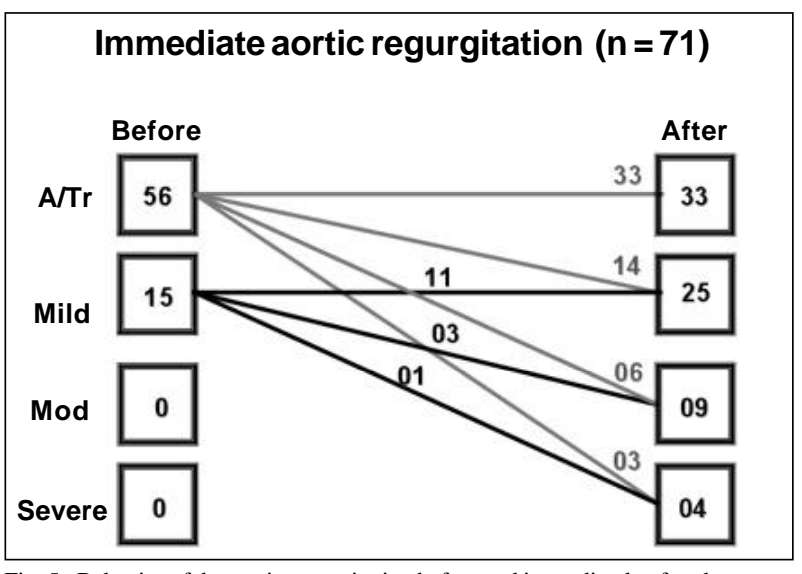

Fig. 5 - Behavior of the aortic regurgitation before and immediately after the procedure. A- absent; Tr- trivial; Mod- moderate.

tion, left ventricular systolic pressure, balloon-ring ratio, thickening degree of the leaflets. However, the use of the carotid route or of adenosine right before dilation had a protective impact on the appearance or worsening of aortic regurgitation (OR-2.2;95\% CI:-1.5-3.2).

In 6 of the 58 patients $(10.3 \%)$ in whom the femoral route was used, loss of the distal pulse was observed. Four of these patients were assisted in the 5 first years of our experience, when low-profile balloons were not yet available on the market. Of those 6 patients, 3 required surgical thrombectomy, which was successfully performed, but 1 patient evolved with discrete motor dysfunction in the limb involved in the procedure. In the 3 remaining patients, although no complete return of the pulse occurred after the usual clinical measures (heparin and streptokinase infusion) were taken, no surgical intervention was necessary due to maintenance of distal perfusion with satisfactory collateral circulation.

Of the 11 neonates/infants undergoing the procedure through the carotid route, 1 died and another had Horner's syndrome, due to difficult percutaneous puncture of the carotid artery. The latter evolved with focal convulsions due to ipsilateral stroke in regard to the site of puncture, with total neurologic and tomographic recovery during the follow-up ${ }^{19}$. In the subsequent patients, in whom the carotid route was achieved through surgical dissection with the introduction of low-profile sheaths under direct visualization, high-quality surgical correction was possible in all cases, and no type of neurological complication occurred. In $7(9.8 \%)$ patients, transfusion of hemoderivates was required. Two patients had transient asystolia after balloon inflation, with the return of normal beats after balloon deflation. In the 4 patients receiving high doses of adenosine, no difficulty in returning to the previous sinus rhythm was observed. In 3 patients, morphology of the left bundlebranch appeared on the electrocardiogram after the procedure and disappeared before hospital discharge. One neonate experienced bronchial aspiration at the hospital, evolving with severe pneumonia and death on the 10th day of hospitalization.
Fifty-five (77.4\%) patients were discharged from the hospital on the day following the procedure. The mean duration of hospitalization varied from 1 to 15 (median of 2) days. Echocardiography performed in 58 patients on the day following the procedure revealed a maximal systolic gradient of $30 \pm 20 \mathrm{mmHg}$. A mean follow-up ranging from 15 days to 131 months (mean of 50.5 \pm 38.4 ; median of 40) was carried out in 37 patients. A young adult with associated aortic and pulmonary restenosis died suddenly 15 days after dilation of both valves.

Restenosis was observed in 6/36 patients (16.6\%), 1 of whom had associated severe aortic regurgitation. Three patients with a peak-to-peak systolic gradient $>40 \mathrm{mmHg}$ right after the procedure developed restenosis in a shorter period of time (14.5+3 months vs $25.5+5$ months; $\mathrm{P}<0.01)$. Of these 6 patients, the 2 with a hypoplastic ring were referred for surgery for valvular replacement with ring widening. One patient with associated severe aortic regurgitation also underwent valvular replacement. Two patients underwent successful redilation with a balloon catheter, and another awaits new dilation. The probability of being restenosis-free was approximately $60 \%$ in a 90 -month follow-up (fig. 6).

During follow-up, aortic regurgitation appeared or worsened in 19 patients $(52.7 \%)$, and it was considered severe in $6(16.6 \%)$ patients. The patients with moderate or severe aortic regurgitation right after the procedure evolved with significant aortic regurgitation and had their surgeries indicated in a shorter period of time $(13.3 \pm 5$ months vs $34.8 \pm$ 10.5 months; $\mathrm{P}<0.01)$. Of 6 patients with significant aortic regurgitation, 4 were referred for valvular replacement (1 with associated restenosis and 2 still await intervention). The probability of being free from significant aortic regurgitation and of requiring intervention was $50 \%$ in a 90 -month follow-up(fig. 7).

A 7-year-old child who had poor oral hygiene, endocarditis, and abscess in the aortic valve 55 months after dilation, required surgical treatment and died in the postoperative period. Twenty-six of the $36(72.2 \%)$ patients undergoing late follow-up were event-free and their maximal systolic gradient on echocardiography was $36+14 \mathrm{mmHg}$. In the patients undergoing recatheterization at the beginning of our study, the peak-to-peak transvalvular gradient was $25.5 \pm 20.8$ $\mathrm{mmHg}$, similar to that found right after dilation $(\mathrm{P}=0.9)$.

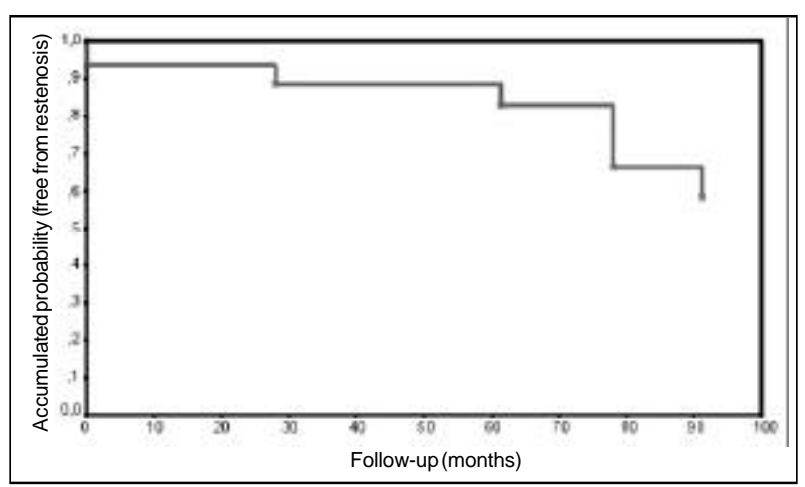

Fig. 6 - Actuarial analysis with estimates of patients free from restenosis. 


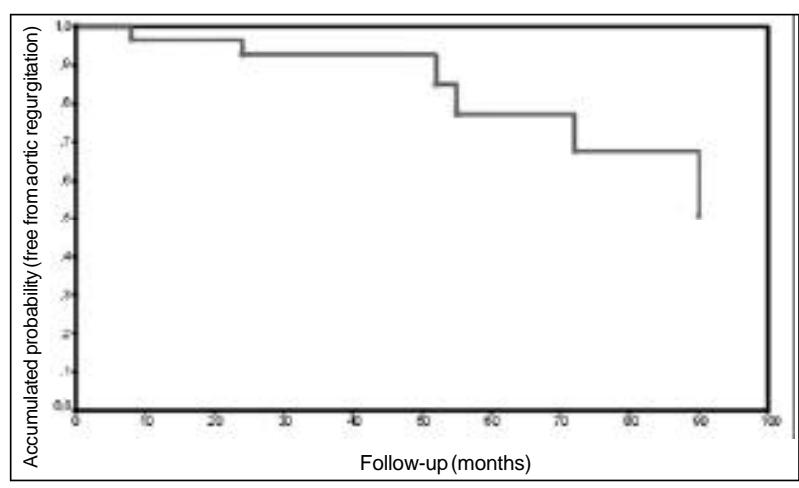

Fig. 7 - Actuarial analysis with estimates of patients free from significant aortic regurgitation.

\section{Discussion}

This study shows that aortic valvuloplasty in aortic stenosis of congenital origin is an effective procedure to reduce the transaortic gradient in different age groups, with acceptable immediate morbidity and mortality and satisfactory results during a midterm follow-up. It has a palliative character, postponing an inevitable surgical valvular intervention. Several points deserve consideration.

The mortality rate observed in this series, although slightly higher than that in other series, is acceptable ${ }^{3-29}$. When the procedure was performed in neonates and infants, mainly in those with ventricular dysfunction, the risk was greater. The death of an 8-year-old child due to occlusion of the coronary artery secondary to dissection caused by manipulation of a Teflon-coated guidewire going through the stenotic valvular orifice could have been avoided with the use of extremely flexible and less traumatic guidewires, such as the hydrophilic coated or the coronary steerable guidewires.

As this case series reflects 15 years of experience with aortic valve dilation, the progressive improvement in the technology of balloon fabrication and the refinement of the technique during this period were factors that introduced inevitable biases in the results. Due to the use of high-profile balloons, the loss of a pulse was more commonly observed in the beginning of our study and sometimes required surgical repair. The appearance of new low-profile balloons substantially reduced the risk of arterial lesion. The use of double balloons ( 1 balloon catheter in each femoral artery) in patients with greater body surface, may also help to reduce the incidence of arterial complications ${ }^{39,40}$. However, the procedure becomes more demanding and requires more time, as observed in this case series, and more than one operator. It is worth noting that basic measures, such as careful arterial puncture reaching only the anterior wall of the vessel, anesthesia/analgesia to prevent arterial spasm, adequate hydration, heating of the limbs during the procedure, liberal use of heparin, and careful hemostatic compression after removal of the introducer should continue to be part of the interventional routine in preventing arterial lesion.

In this study, carotid cut-down not only made the performance of the procedure feasible in neonates and infants ${ }^{43,44}$, but also substantially shortened its time. This is fundamental for this type of high-risk population, which, sometimes, has associated severe ventricular dysfunction. It is worth noting that the performance of the procedure by our team in a preterm neonate weighing $1.1 \mathrm{~kg}$ in 40 minutes resulted in no complications. The multicenter experience shows that the results of aortic valvuloplasty through the carotid route in young infants ( $<6$ months of age $)$ are very good, with high success indices and low complication rates in patients with no risk factors ${ }^{20}$. Imaging studies show that carotid permeability is maintained in more than $95 \%$ of the patients and that neurological complications are exceptional ${ }^{20}$.

The occurrence or progression of aortic regurgitation immediately after dilation is common, but usually of mild magnitude in most cases. No patient in this case series required immediate surgical intervention due to this complication. Although no predictor was found in the multivariate analysis, we believe that the occurrence of aortic regurgitation may be related to the "dance" of the balloon, which is sometimes observed in the left ventricular outflow tract during balloon inflation. As this is a retrospective study, that variable was not directly assessed, because those data were not always documented or recorded for analysis. However, some findings point towards this hypothesis. Due to severe obstruction, ventricular hypertrophy, and consequent hyperdynamic ventricular contractions, the balloon tends to be expelled from the ventricle after its inflation, leading to a push-and-pull movement in the outflow tract, which may injure the leaflets and valvular commissures. We believe that this "dance" may be minimized with some technical maneuvers providing greater balloon stability. In the carotid route, as the distal extremity of the sheath introduced in the carotid artery is located in the ascending aorta, it makes any movement of the balloon difficult after its inflation. The use of adenosine in high doses to induce temporary intense bradycardia or asystolia ${ }^{38}$ may prevent the balloon from being expelled from the left ventricle. These measures were associated with a lower occurrence of aortic regurgitation in this series and were not followed by major complications. For greater stabilization, the interventionist should use the longest balloons possible and extra-stiff guidewires with a large ventricular loop. The progression of long sheaths to the ascending aorta is another technical artifice. The anterograde route, which was not used in this case series, could also have helped in avoiding greater damage to the aortic valve, although it is a more laborious procedure from the technical point of view ${ }^{39}$. In the other aortic valvuloplasty series, appearance or progression of aortic regurgitation was also common. The following factors have been implicated in the occurrence of that finding: the use of balloons with larger dimensions (balloon-ring ratio $>1)^{45}$; ring diameter smaller than $8 \mathrm{~mm}$ and greater than $16 \mathrm{~mm}^{45}$; presence of the degree of regurgitation greater than trivial prior to dilation ${ }^{45}$; and unicommissural valves in neonates ${ }^{6}$.

The lack of follow-up of the entire cohort of our pa- 
tients is a limitation of this study and may have influenced the interpretation of late results. This limitation also occurs in other great Brazilian cardiology centers, whose patients return to their cities of origin, making the obtainment of follow-up data difficult. Even so, we believe that some points deserve further comments. The approximate $17 \%$ restenosis index in a mean 50-month follow-up was similar to that reported in previous publications about balloon catheter valvuloplasty. Similarly to what was found in other case series ${ }^{25-28}$, gradients above $40 \mathrm{mmHg}$ right after valvuloplasty was associated with restenosis in a shorter period of time in our study. Age $<3$ years has also been implicated as a predictor of restenosis ${ }^{25}$. In the absence of contraindications, valvuloplasty may be successfully repeated due to restenosis, as in 2 of our patients and according to the literature $^{46,47}$. Although in our case series aortic valvuloplasty was not performed in cases of restenosis after surgical valvulotomy, the results of this type of approach are as effective as those of native valves and should be applied in that situation $^{48,49}$.

Progression of aortic regurgitation is a frequent and alarming finding in the clinical management of outpatient care unit patients. This observation is universal and common to all percutaneous and surgical case series whose risk factors have not yet been completely identified.

Progression of aortic regurgitation is more related to intrinsic morphological valvular abnormalities than to any other therapeutic procedure per se. This merely reflects the modified natural history of the disease. Considering that both the balloon and the scalpel cause variable degrees of fractures in the sites of commissural fusion ${ }^{50-52}$, which is the major mechanism of stenosis, the progression of the regurgitation is not a surprising finding. In our and other case series ${ }^{25,27,28}$, the progression of aortic regurgitation to moderate or severe degrees was associated with the need for surgery in a shorter period of time. It is worth noting that the presence of isolated severe aortic regurgitation is not per se an indication of valvular surgery, which is one of the most difficult tasks in cardiology practice and should take other factors into consideration. That surgery should be ideally performed with preserved ventricular function or in the early phases of deterioration at the most. As the symptoms appear only in the more advanced phases of ventricular dysfunction, they should not be used as a parameter to guide clinical decisions. Some professionals use the serial analysis of the dimensions of the cardiac area on chest radiography to base their decision. When the area increases, surgery is indicated. Although that is a practical method, we believe that others may provide a greater degree of objectivity and sensitivity for that important decision. In the analysis of the ejection fraction on echocardiography, the end diastolic volume (which is clearly increased in aortic regurgitation) is incorporated into the calculation, and, therefore, the finding of above normal values is the rule in cases of severe regurgitation ${ }^{41,42}$. Therefore, normal or closeto-normal values may denote a reduction in the systolic function and consequent late indication. We believe that the serial measurement of the left ventricular end systolic volume indexed for body surface performed on echocardiography is a very useful parameter for surgical indication. With the different values plotted in a percentile graph in serial form (available in textbooks), it is easier to notice when a change in the curve pattern occurs, indicating a sudden increase in the end systolic volume due to the initial worsening in ventricular function ${ }^{42}$.

Attempts to compare surgical and percutaneous experiences are limited because they do not adjust the innumerous differences existing between the 2 therapeutic approaches, introducing biases in patient selection and time, among other factors ${ }^{53}$. In addition, no uniformity exists in the literature in regard to the definition of restenosis and significant aortic insufficiency. The indications for intervention for restenosis and severe aortic regurgitation also vary extremely among the institutions and in different case series. Because it is a more recent procedure, valvuloplasty comprised a smaller number of patients and a shorter follow-up period in the assessment of the results. The catheterization techniques had a progressive evolution, as did the surgical techniques. In the last few years, the world pediatric cardiology community had a tendency to adopt valvuloplasty as an initial therapeutic method to palliate aortic stenosis; therefore, the comparison of the more current results obtained after percutaneous intervention with the old results after surgical valvulotomy is difficult. In addition, the comparison of results of different institutions is not appropriate at all. Different institutions have different protocols, techniques, strong and weak points, in addition to reflecting individual attitudes, which introduce biases to the analyses. Variable indices of restenosis and functional valvular deterioration with progressive aortic insufficiency are findings common to all surgical and percutaneous case series. Comparisons in multicenter series of contemporary neonatal patients ${ }^{53}$ or in series of contemporary ${ }^{21}$ or not contemporary ${ }^{24}$ patients of single institutions point to overlapping results with the 2 techniques. Therefore, we believe that the decision to refer a patient for balloon valvuloplasty or surgical valvulotomy should be individualized in different institutions according to local characteristics.

Even recognizing the intrinsic difficulties in comparing the results of balloon catheter aortic valvuloplasty with those of surgical valvulotomy, the former provide unequivocal advantages. The shorter period of hospitalization and the lower costs are important features. Absence of a surgical scar is not only a cosmetic advantage. The absence of initial surgical manipulation with preservation of the thoracic cavity represents an enormous benefit for the patient and the surgeon at the time of a future inevitable surgical intervention, it being either valve replacement or the Ross procedure. Absence of fibrosis and adhesions technically facilitates major surgical procedures, helping in improving the results and in minimizing postoperative complications.

Based on data shown here, we believe that aortic valvuloplasty should be offered as the initial therapeutic method to palliate congenital aortic stenosis in services with experience in interventional cardiology for congenital 
heart diseases. That technique has satisfactory short- and midterm results, postponing an inevitable surgical intervention for valvular dysfunction.

Addendum - After finishing this study, aortic valvuloplasty was performed in 3 more patients: 2 neonates approached through the carotid route (one with associated coarctation of the aorta, which was dilated in the same session), in whom the procedure was completed with no complications and without aortic insufficiency. The third patient was 17 years old and underwent successful dilation through the femoral route with the double balloon technique, but did not respond to the administration of high doses of adenosine. Moderate aortic regurgitation appeared. Another patient, who was 12 years old and had undergone valvuloplasty 18 months earlier, underwent the surgical Ross procedure due to progressive aortic regurgitation and deterioration of ventricular function. These findings corroborate the previously cited results and comments.

\section{References}

1. Freedom RM, Mawson JB, Yoo SJ, Benson LN. Left Ventricular Outflow Tract Obstruction. In: Congenital Heart Disease. Textbook of Angiography. Armonk, NY: Futura Publishing Co. Inc., 1997: 787-847.

2. Keane JF, Driscoll DJ, Gersony WM, et al. Second natural history study of congenital heart defects: results of treatment of patients with aortic valvar stenosis. Circulation 1993; 87(Suppl. 2): 16-27.

3. Lababidi Z. Aortic balloon valvuloplasty. Am Heart J 1983; 106: 751-2.

4. Lababidi Z, Wu J, Walls JT. Percutaneous balloon aortic valvuloplasty: results in 23 patients. Am J Cardiol 1984; 54: 194-7.

5. Helgason H, Keane JF, Fellows KE, Kulik TJ, Lock JE. Balloon dilation of the aortic valve.: studies in normal lambs and in children with aortic stenosis. J Am Coll Cardiol 1987; 9: 816-22.

6. Choy M, Beekman RH, Rocchini AP, Crowley DC, Snider AR, Rosenthal A Percutaneous balloon valvuloplasty for valvar aortic stenosis in infants and children. Am J Cardiol 1987; 59: 1010-3.

7. Sholler GF, Keane JF, Perry SB, Sanders SP, Lock JE. Balloon dilation of congenital aortic stenosis: results and influence of technical and morphological features on outcome. Circulation 1988; 78: 351-60.

8. Rao SP, Thapar MK, Wilson AD, Levy JM, Chopra PS. Intermediate-term followup results of balloon valvuloplasty in infants and children with special references to cause of restenosis. Am J Cardiol 1989; 64: 1356-60.

9. Vogel M, Benson L, Burrows P, Smallhorn JF, Freedom RM. Balloon dilation of congenital aortic valve stenosis in infants and children; short and intermediate results. Br Heart J 1989; 62: 148-53.

10. Sullivan ID, Wren C, Bain $\mathrm{H}$, et al. Balloon dilation of aortic valve for congenital aortic stenosis in childhood. Br Heart J 1989; 61: 186-91.

11. Shrivastava S, Das GS, Dev V, Sharma S, Rajani M. Follow-up after percutaneous balloon valvoplasty for noncalcific aortic stenosis. Am J Cardiol 1990; 65: 250-2.

12. Shaddy RE, Boucek MM, Sturttevant JE, Ruttenberg HD, Orsmond GS. Gradient reduction, aortic valve regurgitation and prolapse after balloon aortic valvuloplasty in 32 consecutive patients with congenital aortic stenosis. J Am Coll Cardiol 1990; 16: 451-6.

13. Keane JF, Perry SB, Lock JE. Balloon dilation of congenital valvular aortic stenosis. J Am Coll Cardiol 1990; 16: 457-8.

14. Rocchini AP, Beekman RH, Shachar GB, Benson L, Schwartz D, Kan JS. Balloon aortic valvuloplasty: results of the Valvuloplasty and Angioplasty of Congenital Anomalies Registry. Am J Cardiol 1990; 65: 784-9.

15. O'Connor BK, Beekman RH, Rocchini AP, Rosenthal A. Intermediate-term effectiveness of balloon valvuloplasty for congenital aortic stenosis: a prospective follow-up study. Circulation 1991; 84: 732-8.

16. Witsenburg M, Cromme-Dijkuis AH, Frohn-Mulder IM, Hess J. Short and midterm results of balloon valvuloplasty for valvular aortic stenosis in children. Am J Cardiol 1992; 69: 945-50.

17. Lababidi $Z$, Weinhaus $L$. Successful balloon valvuloplasty for neonatal critical aortic stenosis. Am Heart J 1986; 12: 913-6.

18. Wren C, Sullivan I, Bull C, Deanfield J. Percutaneous balloon dilation of the aortic valve stenosis in neonates and infants. Br Heart J 1987; 58: 608-12.

19. Kasten-Sports CH, Piechaud J, Sidi D, Kachaner J. Percutaneous balloon valvuloplasty in neonates with critical aortic stenosis. J Am Coll Cardiol 1989; 13: 1102-5.

20. Robinson BV, Brzezinska-Rajszys G, Weber H, et al. Balloon aortic valvotomy through a carotid cutdown in infants with severe aortic stenosis: results of the multicenter registry. Cardiol Young 2000; 10: 25-32.

21. Gatzoulis MA, Rigby ML, Shinebourne EA, Redington A. Contemporary results of balloon valvuloplasty and surgical valvotomy for congenital aortic stenosis. Arch Dis Child 1995; 73: 66-9.
22. Moore P, Egito E, Mowrey H, Perry SB, Lock JE, Keane JE. Midterm results of balloon dilation of congenital aortic stenosis: predictors of success. J Am Coll Cardiol 1996; 27: 1257-63.

23. Kuhn MA, Latson LA, Cheatham JP, Fletcher SE, Foreman C. Management of pediatric patients with isolated valvar aortic stenosis by balloon aortic valvuloplasty. Cathet Cardiovasc Diagn 1996; 39: 55-61.

24. Justo RN, McCrindle BW, Benson LN, Williams WG, Freedom RM, Smallhorn JF. Aortic valve regurgitation after surgical versus percutaneous balloon valvotomy for congenital aortic valve stenosis. Am J Cardiol 1996; 77: 1332-8.

25. Galal O, Rao PS, Al-Fadley F, Wilson AD. Follow-up results of balloon aortic valvuloplasty in children with special reference to causes of late aortic insufficiency. Am Heart J 1997; 133: 418-27.

26. Borghi A, Agnoletti G, Valsecchi O, Carminati M. Aortic balloon dilatation for congenital aortic stenosis: report of 90 cases (1986-98). Heart 1999; 82: 10.

27. Demkow M, Ruzyllo W, Ksiezycka E, et al. Long-term follow-up results of balloon valvuloplasty for congenital aortic stenosis: predictors of late outcome. J Inv Cardiol 1999; 11: 220-6.

28. Jindal RC, Saxena A, Juneja R, Kothari SS, Shrivastava S. Long-term results of balloon aortic valvotomy for congenital aortic stenosis in children and adolescents. J Heart Valve Dis 2000; 9: 623-8.

29. Rosenfeld HM, Landzberg MJ, Perry SB, Colan SD, Keane JF, Lock JE. Balloon aortic valvuloplasty in the young adult with congenital aortic stenosis. Am J Cardiol 1994; 73: 1112-7.

30. Rao PS. Balloon valvuloplasty for aortic stenosis. In: Rao PS, ed. Transcatheter Therapy in Pediatric Cardiology. New York: Wiley-Liss, 1993: 105-27.

31. Yeager SB, Flanagan MF, Keane JF. Catheter interventions: balloon valvotomy. In: Lock JE, Keane JF, Perry SB, et al, eds. Diagnostic and Interventional Catheterization in Congenital Heart Disease. Boston: Kluwer Academic Publishers, 2000: 151-78.

32. Beekman RH, Rocchini AP, Gilon JH, Mancini J. Hemodynamic determinants of the peak systolic left ventricular-aortic pressure gradient in children with valvar aortic stenosis. Am J Cardiol 1992; 69: 813-5.

33. Lima VC, Zahn E, Houde C, Smallhorn J, Freedom RM, Benson LN. Non-invasive determination of the systolic peak-to-peak gradient in children with aortic stenosis: validation of a mathematical model. Cardiol Young 2000; 10: 115-9.

34. Rhodes LA, Colan SD, Perry SB, Jonas RA, Sanders SP. Predictors of survival in neonates with critical aortic stenosis. Circulation 1991; 84: 2325-35.

35. Leung MP, McKay R, Smith A, Anderson RH, Arnold R. Critical aortic stenosis in early infancy: anatomic and echocardiographic substrates of successful open valvotomy. J Thorac Cardiovasc Surg 1991; 101: 525-6.

36. Lofland GK, McCrindle BW, Williams WG, et al. Critical aortic stenosis in the neonate: a multi-institutional study of management, outcomes and risk factors. Congenital Heart Surgeons Society. J Thorac Cardiovasc Surg 2001; 121: 10-27.

37. Pedra CAC, Sousa LN, Fontes VF. Estenose aórtica e persistência do canal arterial. Importância da oclusão do canal para correta determinação do gradiente aórtico. Rev Bras Cardiol Inv 2000; 8: 24-7.

38. De Giovanni JV, Edgar RA, Cranston A. Adenosine-induced transient cardiac standstill in catheter interventional procedures for congenital heart disease. Heart 1998; 80: 330-3.

39. Beekman RH, Rocchini AP, Crowley DC, et al. Comparison of single and double balloon valvuloplasty in children with aortic stenosis. J Am Coll Cardiol 1988; 12: 480-5.

40. Yeager SB. Balloon selection for double balloon valvotomy. J Am Coll Cardiol 1987; 9: 467.

41. Snider AR, Serwer GA, Ritter SB. Methods of obtaining quantitative information from the echocardiographic examination. In: Echocardiography in Pediatric Heart Disease. St Louis: Mosby, 1997: 180-6.

42. Snider AR, Serwer GA, Ritter SB. Abnormalities of the left ventricular outflow. In: 
Echocardiography in Pediatric Heart Disease. St Louis: Mosby, 1997; 439-44.

43. Pedra CAC, Pedra SRFF, Fontes VF. Estenose aórtica crítica. In: Santana MVT. Ed. Cardiopatias Congênitas no Recém Nascido. Diagnóstico e Tratamento. São Paulo: Atheneu; 2000: 309-17.

44. Magee AG, Nykanen D, McCRindle BW, Wax D, Freedom RM, Benson LN Balloon dilation of severe aortic stenosis in the neonate: comparison of anterograde and retrograde catheter approaches. J Am Coll Cardiol 1997; 30: 1061-6.

45. McCrindle BW for the VACA Registry Investigators. Independent predictors of immediate results of percutaneous balloon aortic valvotomy in childhood. Am J Cardiol 1996; 77: 286-93.

46. Shim D, Lloyd TR, Beekman RH III. Usefulness of repeat balloon aortic valvuloplasty in children. Am J Cardiol 1997; 79: 1141-6.

47. Satou GM, Perry SB, Lock JE, Piercey GE, Keane JF. Repeat balloon dilation of congenital valvar aortic stenosis: immediate results and midterm outcome. Cathet Cardiovasc Diagn 1999; 47: 47-51.

48. Sreeram N, Kitchiner D, Williams D, Jackson M. Balloon dilation of the aortic valve after previous surgical valvotomy: immediate and follow-up results. $\mathrm{Br}$ Heart J 1994; 71: 558-60.

49. Meliones JN, Beekman RH, Rocchini AP, Lacina SJ. Balloon valvuloplasty for recurrent aortic stenosis after surgical valvotomy in childhood: immediate and follow-up studies. J Am Coll Cardiol 1989; 13: 1106-10.

50. Phillips RR, Gerlis LM, Wilson N, Walker DR. Aortic valve damage caused by operative balloon dilatation of critical aortic valve stenosis. Br Heart J 1987; 57: 168-0.

51. Waller BF, McKay C, VanTassel JW, Taliercio C, Howard J, Green F. Catheter balloon valvuloplasty of stenotic aortic valves. Part I. Anatomic basis and mechanism of balloon dilation. Clin Cardiol 1991; 14: 836-46.

52. Solymar L, Sudow G, Berggren H, Erikson B. Balloon dilation of stenotic aortic valve in children: an intraoperative study. J Thorac Cardiovasc Surg 1992; 104: 1709-13.

53. Mc Crindle BW, Blackstone EH, Williams WG, et al. Are outcomes of surgical versus transcatheter balloon valvotomy equivalent in neonatal aortic stenosis? Circulation 2001; 104: I 152-8 